\title{
Impact of vitamin E-blended UHMWPE wear particles on the osseous microenvironment in polyethylene particle-induced osteolysis
}

\author{
CARL NEUERBURG ${ }^{1}$, THERESA LOER ${ }^{2}$, LENA MITTLMEIER ${ }^{1}$, CHRISTINA POLAN $^{2}$, ZSUZSANNA FARKAS $^{1}$, \\ LESCA MIRIAM HOLDT $^{3}$, SANDRA UTZSCHNEIDER ${ }^{4}$, JENS SCHWIESAU ${ }^{4,5}$, THOMAS M. GRUPP ${ }^{4,5}$, \\ WOLFGANG BÖCKER ${ }^{1}$, ATTILA ASZODI ${ }^{1}$, CHRISTIAN WEDEMEYER $^{6 *}$ and CHRISTIAN KAMMERLANDER ${ }^{1,7 *}$ \\ ${ }^{1}$ Experimental Surgery and Regenerative Medicine, Department of General, Trauma and Reconstructive Surgery, \\ Munich University Hospital LMU, Munich; ${ }^{2}$ Department of Orthopedics and Trauma Surgery, University Hospital, \\ University of Duisburg-Essen, Essen; ${ }^{3}$ Institute of Laboratory Medicine, Ludwig-Maximilians-University Munich, Munich; \\ ${ }^{4}$ Department of Orthopaedic Surgery, Physical Medicine and Rehabilitation, Munich University Hospital LMU, Munich; \\ ${ }^{5}$ Aesculap AG, Research and Development, Tuttlingen; ${ }^{6}$ KKEL St. Barbara Hospital, Department of Orthopedics and \\ Trauma Surgery, Gladbeck, Germany; ${ }^{7}$ Department of Trauma Surgery, Medical University, Innsbruck, Austria
}

Received July 5, 2016; Accepted September 23, 2016

DOI: $10.3892 / \mathrm{ijmm} .2016 .2780$

\begin{abstract}
Aseptic loosening mediated by wear particleinduced osteolysis (PIO) remains the major cause of implant loosening in endoprosthetic surgery. The development of new vitamin $\mathrm{E}$ ( $\alpha$-tocopherol)-blended ultra-high molecular weight polyethylene (VE-UHMWPE) with increased oxidation resistance and improved mechanical properties has raised hopes. Furthermore, regenerative approaches may be opened, as vitamin E supplementation has shown neuroprotective characteristics mediated via calcitonin gene-related peptide (CGRP), which is known to affect bone remodeling in PIO. Therefore, the present study aimed to further clarify the impact of VE-UHMWPE wear particles on the osseous microenvironment and to identify the potential modulatory pathways involved. Using an established murine calvaria model, mice were subjected to sham operation (SHAM group), or treated with UHMWPE or VE-UHMWPE particles for different experimental durations ( 7,14 and 28 days; $n=6 /$ group). Morphometric analysis by micro-computed tomography detected signifi-
\end{abstract}

Correspondence to: Dr Carl Neuerburg or Dr Christian Kammerlander, Experimental Surgery and Regenerative Medicine, Department of General, Trauma and Reconstructive Surgery, Campus Grosshadern, Munich University Hospital LMU, Marchioninistrasse 15, D-81377 Munich, Germany

E-mail: carl.neuerburg@med.uni-muenchen.de

E-mail: christian.kammerlander@med.uni-muenchen.de

*Contributed equally

Key words: particle-induced osteolysis, vitamin E, $\alpha$-tocopherol, calcitonin gene-related peptide, arthroplasty, murine calvaria cant $(\mathrm{p}<0.01)$ and comparable signs of PIO in all particle-treated groups, whereas markers of inflammation [tumor necrosis factor (TNF)- $\alpha /$ tartrate resistant acid phosphatase (TRAP) staining] and bone remodeling [Dickkopf-related protein 1 (DKK-1)/osteoprotegerin (OPG)] were most affected in the early stages following surgery. Taking the present data into account, VE-UHMWPE appears to have a promising biocompatibility and increased ageing resistance. According to the $\alpha$-CGRP serum levels and immunohistochemistry, the impact of vitamin $\mathrm{E}$ on neuropeptidergic signaling and its chance for regenerative approaches requires further investigation.

\section{Introduction}

Endoprosthetic surgery in orthopedic and trauma patients is known to be one of the mostsuccessful reconstructive approaches, yet wear particle-induced osteolysis (PIO)-mediated by ultrahigh molecular weight polyethylene (UHMWPE) particles remains the major cause of implant loosening (1-3). Depending on the composition of the bearing couple, macrophages phagocytose released wear particles, which leads to an aseptic inflammatory response and subsequently induces osteolysis in the bone-implant interface (4-6). In order to face the problem of PIO, different approaches have been introduced.

In the field of biotribology, major improvements of endoprosthetic components have been presented in the past as an attempt to reduce wear and osteolysis. Thus, the development of new implant designs, materials, sterilisation techniques, oxidation resistance and articulating surface treatments have contributed to a reduction of wear, delamination and structural fatigue (7). As shown in hip and knee simulator studies, the development of improved inserts from conventional UHMWPE towards highly cross-linked UHMWPE is associated with a significant reduction of wear, while clinical investigations have observed reduced osteolysis $(8,9)$. 
The incorporation of vitamin $\mathrm{E}$ ( $\alpha$-tocopherol) into UHMWPE (VE-UHMWPE) either by diffusion following irradiation or blending followed by irradiation is a novel modification made in an aim to increase oxidation resistance and improve the mechanical properties of UHMWPE $(10,11)$. As described previously, vitamin $\mathrm{E}$ has a positive impact in the process of ageing of UHMWPE which is related to a chemical reaction cascade between the macromolecules and oxygen. Irradiation processes from sterilisation or crosslinking generate free bonds (radicals) on the molecules which react with oxygen. One possible outcome of these reaction cascades is chain scission of the macromolecules, leading to mechanical property degradation. Vitamin E can donate hydrogen to react with the free bonds and interrupt this reaction cascade (12).

In a recent study, vitamin E supplementation was shown to have neuroprotective characteristics mediated via calcitonin gene-related peptide (CGRP) nerve fibers which can be of interest (13). Neuropeptides, such as $\alpha$-CGRP have also been detected in the synovial fluid and periarticular tissue of loosened implants which strengthens the impact of the local neurogenic environment in the process of osteolysis and may open new regenerative therapy approaches $(14,15) . \alpha$-CGRP belongs to the calcitonin (CT) peptide family which is a generated analog to calcitonin by alternative splicing of the CALCA gene (16). Previous experiments using knockout mouse models with isolated or combined deficiency of CT/ $\alpha$-CGRP have provided further insight on the impact of the CT family peptides, whereas $\alpha$-CGRP turned out to play an important role in the process of UHMWPE particle induced osteolysis (17-19).

Therefore, the aim of the present study was to further clarify the impact of VE-UHMWPE wear particles on the osseous microenvironment of an established murine calvaria model and to identify the potential modulatory pathways.

\section{Materials and methods}

Animals. All experiments were performed and registered in accordance to the local authorities (Reference nos. 55.2-1-542532-232-2013) similar to the NIH guidelines for the care and use of laboratory animals (NIH Publication \#85-23 Rev. 1985). As described previously, a murine calvaria model of UHMWPE particle-induced osteolysis was established using 54 male C57BL/6 mice provided by Charles River Laboratories, Inc. (Sulzfeld, Germany) $(17,18)$. The animals were delivered at the age of 10 weeks and surgery was performed at the age of 12 weeks. The mice were divided into 3 groups as follows: one group was subjected to sham operation (SHAM group), the animals in the second group were treated with UHMWPE particles and the animals in the third group were treated with vitamin E-blended UHMWPE (VE-UHMWPE) particles. Furthermore, the animals were divided into subgroups according to the different durations of the experimental duration (7, 14 and 28 days). Thus, each group consisted of 6 mice. During the experimental period, food (V 1534-300; Ssniff Spezialdiäten GmbH, Soest, Germany) and water was supplied ad libitum. The animals were kept under specific pathogen-free (SPF) conditions. Post-operative analgesia was achieved using metamizole dissolved in the animals' drinking water $(1.2 \mathrm{mg} / \mathrm{ml})$. The animals were sacrificed at the end of the experimental duration, depending on the group.
Particles. The treated mice in the second group received conventional UHWMPE particles, whereas the animals in the third group received crosslinked VE-UHMWPE particles obtained from Aesculap (Tuttlingen, Germany) and generated as previously described (20). The equivalent circle diameter (ECD) of the UHMWPE particles [detected according to ASTM F 1877 (21)] was $1 \mu \mathrm{m}$, whereas $90 \%$ of the particles had a size $<2 \mu \mathrm{m}$. Testing for cytotoxicity and endotoxin purity was performed according to the United States Pharmacopeial Convention (USP) given an endotoxin level <20.0 USP endotoxins per device and no cytotoxic reactivity.

Surgical procedure. All mice were anaesthetised by an intraperitoneal injection of fentanyl $[0.05 \mathrm{mg} / \mathrm{kg}$ body weight $(\mathrm{BW})]$, midazolam (5 mg/kg BW) and medetomidine $(0.5 \mathrm{mg} / \mathrm{kg} \mathrm{BW})$. After shaving the head and sterile draping, a $1 \mathrm{~cm}$ skin incision over the calvarian sagittal midline suture was made and a $1 \times 1 \mathrm{~cm}$ area of the periosteum was exposed and left intact as described previously (19). According to the experimental design, the animals in group A were subjected to sham operation only (SHAM group), the animals in group B received $\sim 30 \mu \mathrm{l}\left(2 \times 10^{8}\right.$ particles/1,000 $\left.\mu \mathrm{l}\right)$ of dried UHMWPE particles implanted periostally, and the animals in group $\mathrm{C}$ received an equivalent amount of VE-UHMWPE wear particles. The incision was closed using a 4-0 Ethilon skin suture (Ethicon, Sommerville, NJ, USA). The animals were sacrificed in a $\mathrm{CO}_{2}$ chamber following an experimental period of 7, 14 or 28 days according to their group.

Micro-computed tomography $(\mu-C T)$. Microstructural differences of the different calvaria were assessed using high-resolution $\mu$-CT (SkyScan 1072; SkyScan, Aartselaar, Belgium). Following sacrifice, the mouse skulls were separated using a sharp cutter at the neck for decapitation and fixed in $4 \%$ paraformaldehyde for $24 \mathrm{~h}$ and stored in $70 \%$ of ethanol thereafter. During scanning, the skulls were placed in a tightly fitting rigid plastic tube inside the scanner's chamber to avoid artifacts. With a resolution set at $19 \mu \mathrm{m}$ at a source voltage of $80 \mathrm{kV}$ and $100 \mu \mathrm{A}$, scanning was performed with rotation of the specimens in equiangular steps of $0.9^{\circ}$. Threedimensional images were computed using the program Cone-beam Reconstruction (SkyScan). Quantitative analysis was performed using CT Analyser (CTAn; SkyScan) with a fixed volume of interest (VOI) having set a rectangular region of interest (ROI) of $4 \times 4 \mathrm{~mm}$ centering the midline suture in the 2D-reconstructed cross-sectional slices. Within the VOI, the parameters of the osseous micro-architecture (BV/TV) were obtained as previously described (22). Three-dimensional images of the calvaria were generated using CT Vol (SkyScan).

Histological analysis. After $\mu$-CT analysis, the decapitated skulls were prepared for histological processing; therefore, the calvaria were removed as an elliptical plate of bone defined by the foramen magnum, auditory canals and orbits. The scalp was kept in order to protect the calvaria in the operated area. The samples were then dehydrated in a graded alcohol series and embedded in a plastic embedding system based on methyl methacrylate (MMA) 'Technovit-9100' (Heraeus-Kulzer, Wehrheim, Germany). Following complete polymerization, the samples were cut using a 'charly' diamond saw (Walter Messner GmbH, 
Norderstedt, Germany) in the coronar plane of the calvaria. Of these samples, 5 - $\mu \mathrm{m}$-thick slices were cut using a Reichert-Jung rotary microtome (Reichert-Jung, Nussloch, Germany).

Masson-Goldner trichrome and toluidine blue staining were performed (Carl Roth GmbH, Karlsruhe, Germany) according to standard protocols. Tartrate resistant acid phosphatase (TRAP) staining was completed with a Napthol AS-BI phosphoric acid solution (N-2250; Sigma-Aldrich Chemie GmbH, Munich, Germany) on randomly selected slices from each group. Osteoclasts were detected in a set field of view following digital photography at a magnification of $\mathrm{x} 10$ with the midline suture in its center (Axio Observer Z.1 AX10, connected to ZEN Imaging Software; Carl Zeiss Microscopy GmbH, Jena, Germany). Immunohistochemistry was performed using canine antimouse monoclonal tumor necrosis factor (TNF)- $\alpha$-antibodies (Cat. no. 17590-1-AP; Acris Antibodies GmbH, Herford, Germany) in a first step conjugated with anti-canine peroxidase polymer in a second step for $30 \mathrm{~min}$ (Medac $\mathrm{GmbH}$, Wedel, Germany). Color development was performed using the chromogen Bright DAB substrate kit (Immunologic, Duiven, The Netherlands) for $10 \mathrm{~min}$. Counterstaining was performed with Mayer's hemalum solution for 5 min (Leica, Wetzlar, Germany). For $\alpha$-CGRP antibody staining, canine anti-mouse polyclonal antibodies were used for $30 \mathrm{~min}$ (Cat. no. A78-128; Antibodies-online $\mathrm{GmbH}$, Aachen, Germany) conjugated with anti-canine peroxidase polymer in a second step as stated above (Cat. no. 414141F; Medac GmbH). Similarly, counterstaining was performed with Mayer's hemalum solution (Leica). Dehydration was performed using a graded alcohol series. To rule out a potential cross-reactivity of the secondary antibody $(\alpha-\mathrm{CGRP} / \mathrm{TNF}-\alpha)$ negative controls were generated with a dilution medium. Similarly, a positive control was prepared for each antibody using spleen samples of mouse cadavers.

Serum analyses. To rule out bone metabolic disorders, retroorbital blood collection was performed at the beginning of the experimental period and calcium, phosphate and alkaline phosphatase levels were quantified. Prior to sacrifice, additional blood samples were taken via puncture of the vena cava. The serum samples were centrifuged at 8,000 x g for $10 \mathrm{~min}$, aliquoted and frozen at $-70^{\circ} \mathrm{C}$.

Biomarker analysis was performed according to the manufacturer's instructions. Thus, serum $\alpha$-CGRP levels were detected using a specific enzyme-linked immunosorbent assay (ELISA) kit provided by MyBioSource Inc. (MBS 721907; San Diego, CA, USA). As a marker of bone resorption, Dickkopf-1 (DKK-1) levels were detected using a DKK-1 Quantikine ELISA kit (R\&D Systems, Minneapolis, MN, USA). The serum levels of osteoprotegerin (OPG), which is a marker of bone formation, were detected using a TNFRSF11B Quantikine ELISA kit (R\&D Systems, Minneapolis, MN, USA).

Statistical analysis. Data are reported as the means \pm standard deviation (SD). Normally distributed results were analysed by one-way analysis of variance (ANOVA) to identify global significant differences between groups, followed by post-hoc analysis via Tukey's test or Games-Howell test. In the case of significant variance of the normal distribution, non-parametric tests were applied using the method of Kruskal-Wallis. For statistical analysis, SPSS 22 (IBM Germany GmbH, Ehningen,
Germany) was used and the level of significance was set at $\mathrm{p}<0.05$. Graphics were created with GraphPad Prism 5.0 (GraphPad Software, Inc., La Jolla, CA, USA).

\section{Results}

Apart from 2 mice that suffered cardiovascular arrest under anesthesia, all animals tolerated the surgery and the experimental duration well.

$\mu$-CT. Comparison of the calvarias' histomorphometry within the mice in the SHAM group revealed no relevant differences in bone volume density (BV/TV) between the different experimental duration times of 7, 14 or 28 days (Fig. 1). The intragroup comparison in the UHMWPE-treated mice revealed the smallest BV/TV in the mice within the 7-day experimental duration group, whereas there was a trend towards an increase in $\mathrm{BV} / \mathrm{TV}$ when comparing the UHMWPE-treated mice at 7 and 14 days $(\mathrm{p}=0.101)$ and a significant increase in $\mathrm{BV} /$ TV in mice treated with the UHMWPE particles for 28 days compared to the animals within the 7-day experimental duration group $(p<0.001)$. Similarly, the animals treated with the VE-UHMWPE particles (VE-UHMWPE) showed the lowest $\mathrm{BV} / \mathrm{TV}$ in mice observed at 7 days, whereas there was a trend indicating an increase in BV/TV when comparing the mice treated for 7 days with those treated for 14 days $(p=0.617)$, and a comparison of the animals treated with the VE-UHMWPE particles for 7 days with those treated for 28 days showed a significant increase in BV/TV $(\mathrm{p}<0.001)$.

Intergroup comparisons of $\mathrm{BV} / \mathrm{TV}$ (Fig. 1) revealed a significantly reduced BV/TV in the mice treated with either the UHMWPE or VE-UHMWPE particles for 7 days compared to the mice in the SHAM group $(\mathrm{p}<0.001)$. No significant difference in BV/TV was observed when comparing the UHMWPE particle- with the VE-UHMWPE particle- treated mice at 7 days $(p=0.960)$. At 14 days, there was still a reduction in BV/TV in the UHMWPE particle-treated mice $(\mathrm{p}=0.227)$ and VE-UHMWPE particle-treated mice $(\mathrm{p}=0.008)$ compared to the mice in the SHAM group. Whereas after a period of 28 days, the UHMWPE or VE-UHMWPE particle-treated mice showed a significant increase in BV/TV compared to the mice in the SHAM group (vs. UHMWPE, $\mathrm{p}=0.007$; vs. VE-UHMWPE, $p=0.018)$.

Inflammation and osteoclast activity. Immunohistochemistry revealed an increased TNF- $\alpha$ concentration in all mice in the 7 -day experimental duration group compared those in the 28-day experimental duration group. TNF- $\alpha$ antibody staining was most intense at the surface of the calvaria in the area of the midline-suture, and there were no distinct inter- or intradifferences observed in a comparison of either the animals in the SHAM group or the particle treated animals (Fig. 2).

Similar to the TNF- $\alpha$ immunoreactivity, there were considerably more osteoclasts detected by TRAP staining (Fig. 3B) in all animals either in the SHAM group, or those treated with the UHMWPE or VE-UHMWPE particles at 7 days compared to the animals treated at 28 days (Fig. 3C). Intragroup comparison revealed a clear reduction in TRAP-positive cells after 14 days in the mice in the SHAM group and the UHMWPE particle-treated mice compared to their corresponding groups at 7 days, whereas 

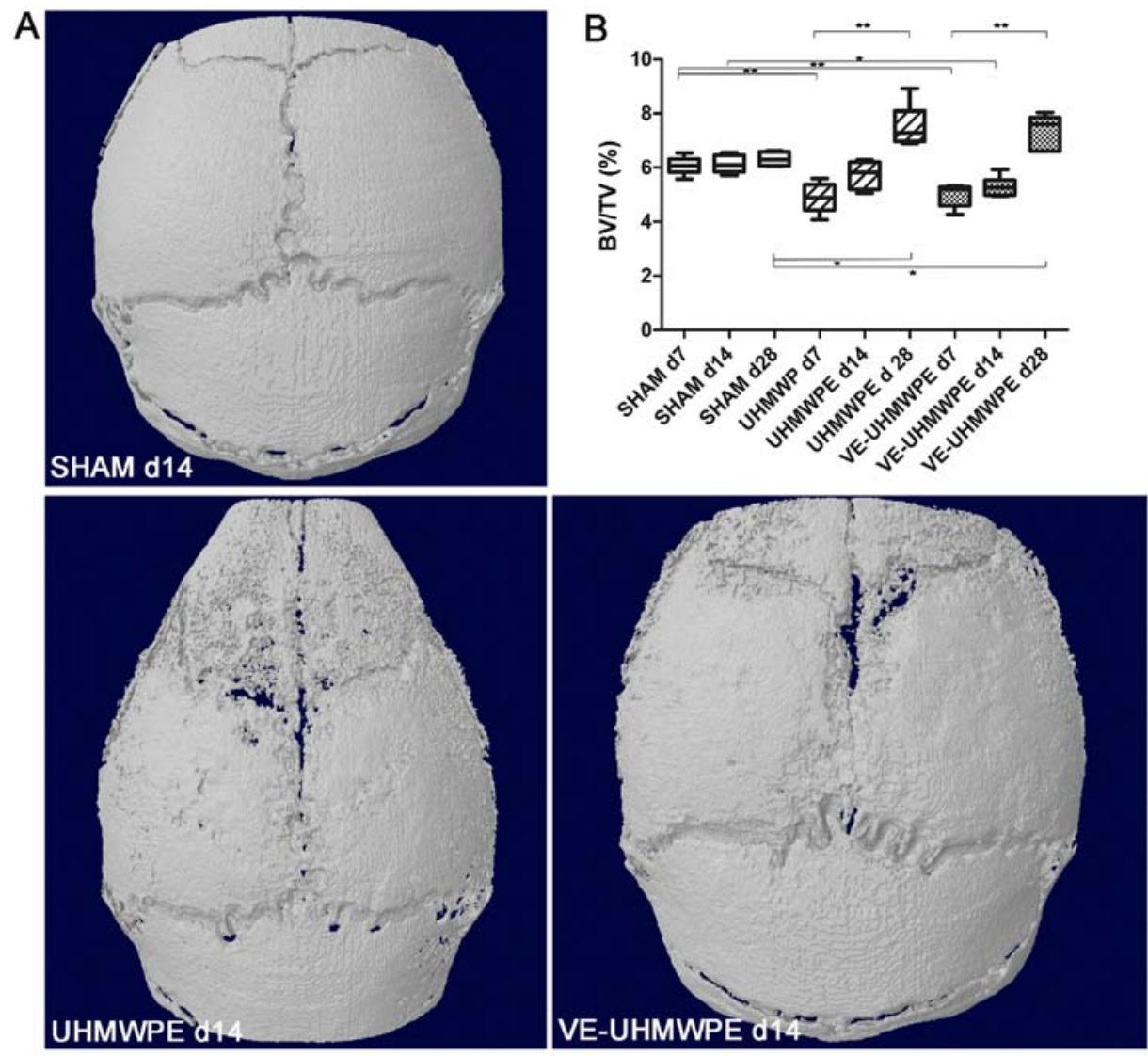

Figure 1. (A) Representative three-dimensional $\mu$-CT reconstructions of the calvaria of mice subjected to sham operation (SHAM) or mice treated with conventional ultra-high molecular weight polyethylene (UHMWPE) particles or vitamin E ( $\alpha$-tocopherol)-blended UHMWPE (VE-UHMWPE) particles following an experimental duration of 14 days. (B) Boxplots demonstrate the mean bone volume density (BV/TV) and standard deviation (SD) detected by $\mu$-CT analysis for each group at 7,14 and 28 days post-surgery $\left(" \mathrm{p}<0.01\right.$ and $\left.{ }^{* *} \mathrm{p}<0.001\right)$. d, day.
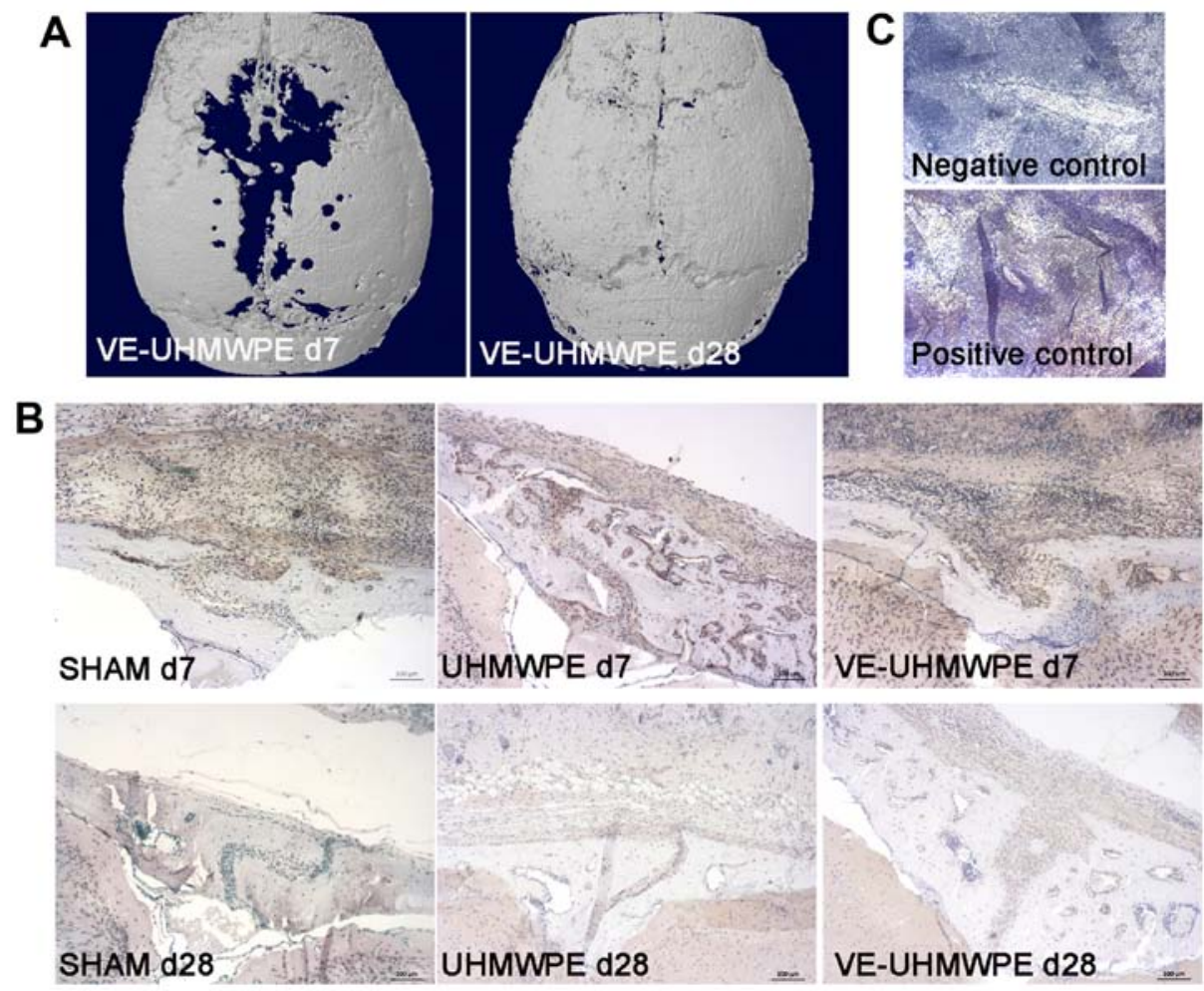

Figure 2. (A) Three-dimensional $\mu$-CT reconstructions of the calvarias of mice treated with vitamin $\mathrm{E}$ ( $\alpha$-tocopherol)-blended ultra-high molecular weight polyethylene (VE-UHMWPE) particles at 7 and 28 days. (B) Representative tumor necrosis factor (TNF)- $\alpha$ antibody-stained mouse calvarial sections following an experimental duration time of 7 and 28 days. Magnification set at x10, the midline suture is located in the center. (C) Negative and positive controls of the spleen were included. d, day. 

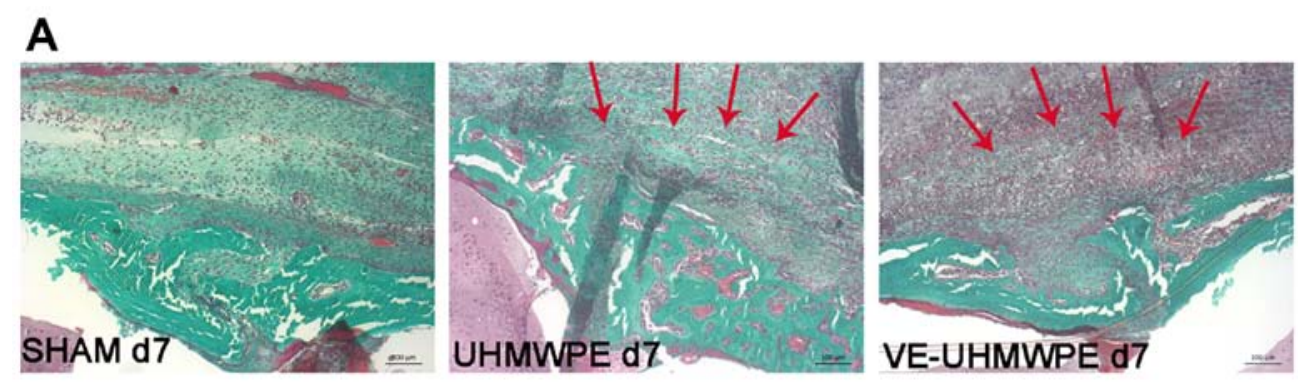

B
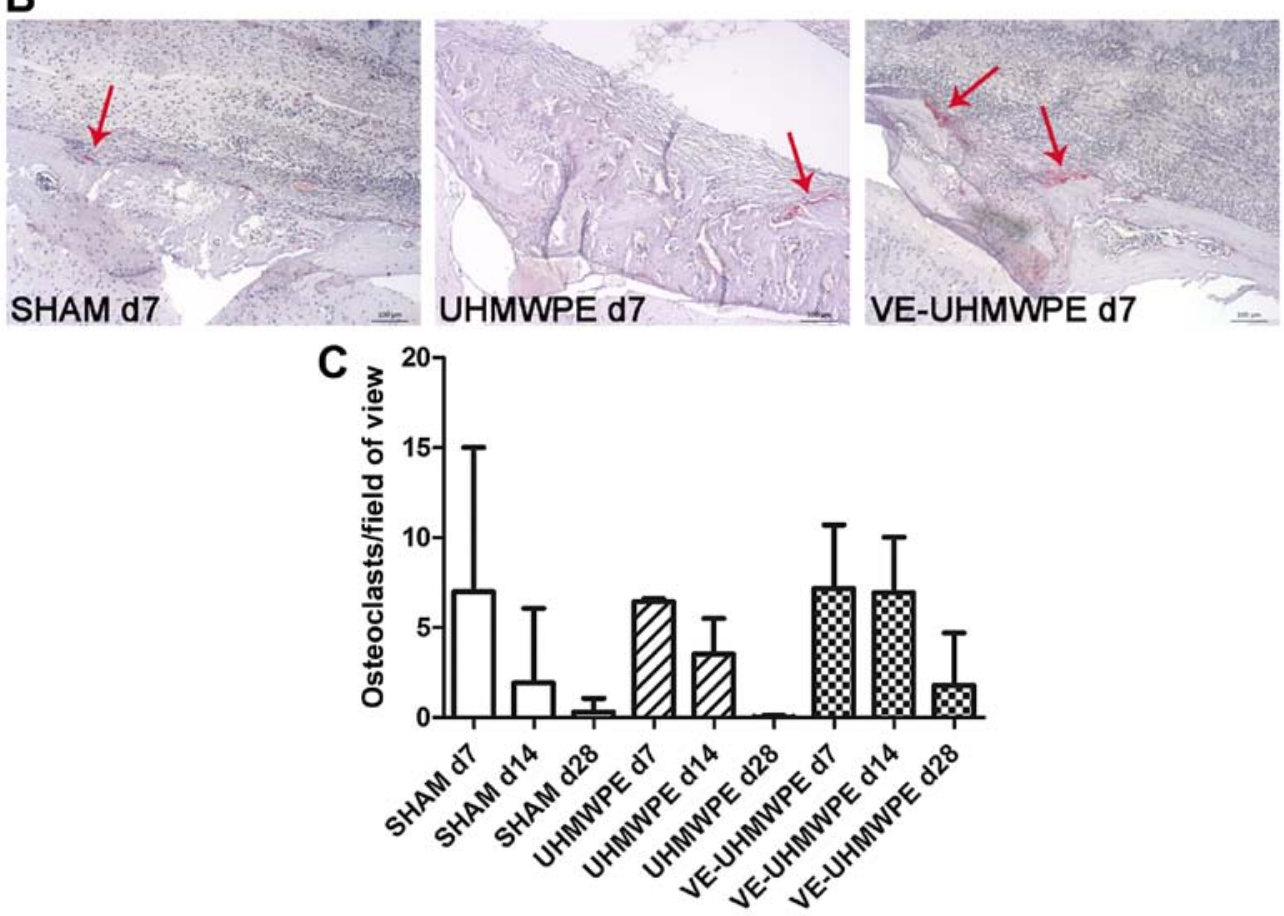

Figure 3. (A) Masson-Goldner staining demonstrating the inflammatory response which is most distinct in the animals at 7 days. Arrows indicate the granulomatous tissue. (B) Tartrate resistant acid phosphatase (TRAP) staining demonstrates osteoclasts (arrows) detected in the area of the midline suture as shown in representative histological sections of mice at 7 days. (C) The graph illustrates the mean osteoclast count + SD within a field of view at at $x 10$ magnification. $d$, day.

a clear reduction in TRAP-positive cells in the VE-UHMWPE particle-treated mice was first observed after an experimental time of 28 days, although the SD was relatively large.

Histological analysis of the soft tissues surrounding the midline suture was associated with an increased inflammatory response which was most obvious in the mice in the 7-day experimental duration group, as indicated by the granulomatous tissue on Masson-Goldner-stained sections (Fig. 3A).

a-CGRP-mediated local neurogenic microenvironment. Biomarker analysis of the serum revealed an increase in the $\alpha$-CGRP levels in all groups given a longer experimental time (Fig. 4A). In the UHMWPE and VE-UHMWPE particletreated mice, the detected $\alpha$-CGRP levels were significantly higher at 28 days compared to those at 14 days $(p=0.036$ and $\mathrm{p}<0.001)$. Intergroup comparisons only revealed significantly higher $\alpha$-CGRP levels in the mice treated with the VE-UHMWPE particles for 28 days compared to the corresponding controls (SHAM group; $\mathrm{p}=0.009$ ).

According to immunohistochemistry, however, $\alpha$-CGRP antibody staining revealed a more distinct $\alpha$-CGRP concen- tration in the UHMWPE and VE-UHMWPE particle-treated groups after a period of 14 days compared to their counterparts with an experimental time of 7 days (Fig. 4B).

Biochemical markers of bone turnover. Bone metabolic disorders were excluded at the beginning of the experimental duration time by the detection of calcium, phosphate and alkaline phosphatase levels. Following dilution with saline in a ratio of $1: 2$, the mean calcium level detected in serum at the beginning of the experimental time was $0.74+0.08 \mathrm{mmol} / \mathrm{l}$, the mean phosphate level was $2.1+0.3 \mathrm{mg} / \mathrm{dl}$ and the mean alkaline phosphatase level was $30.08+4.7 \mathrm{U} / 1$. There were no relevant inter- or intragroup differences observed in any of the animals at the beginning of the experimental duration time (data not shown).

At the end of the experimental time, marker analysis for bone resorption revealed significantly elevated DKK-1 levels in the mice in the SHAM group and the UHMWPE particletreated animals at the experimental time of 7 days compared to their corresponding groups at 14 days $(\mathrm{p}<0.05)$, though no significant differences were observed in a similar comparison 

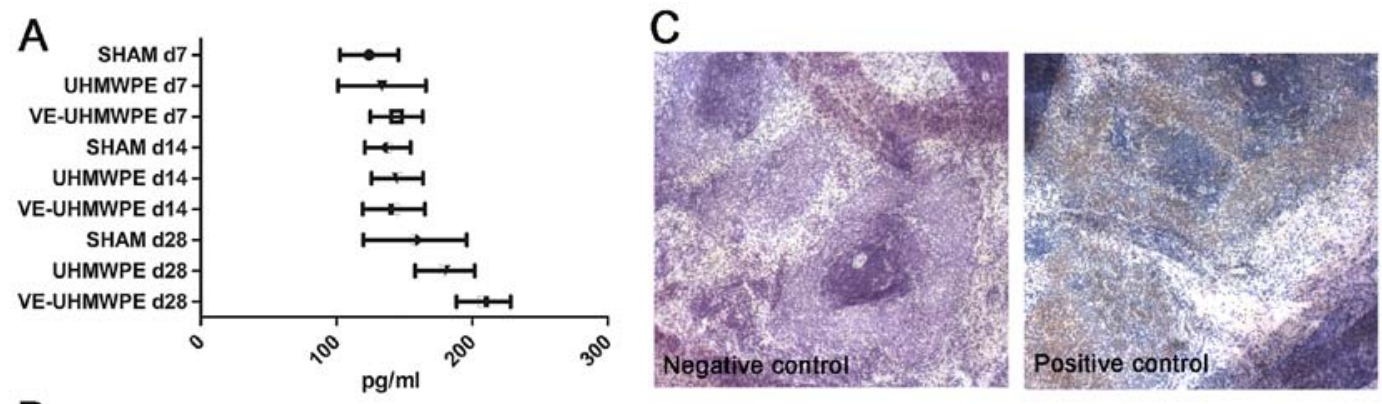

B

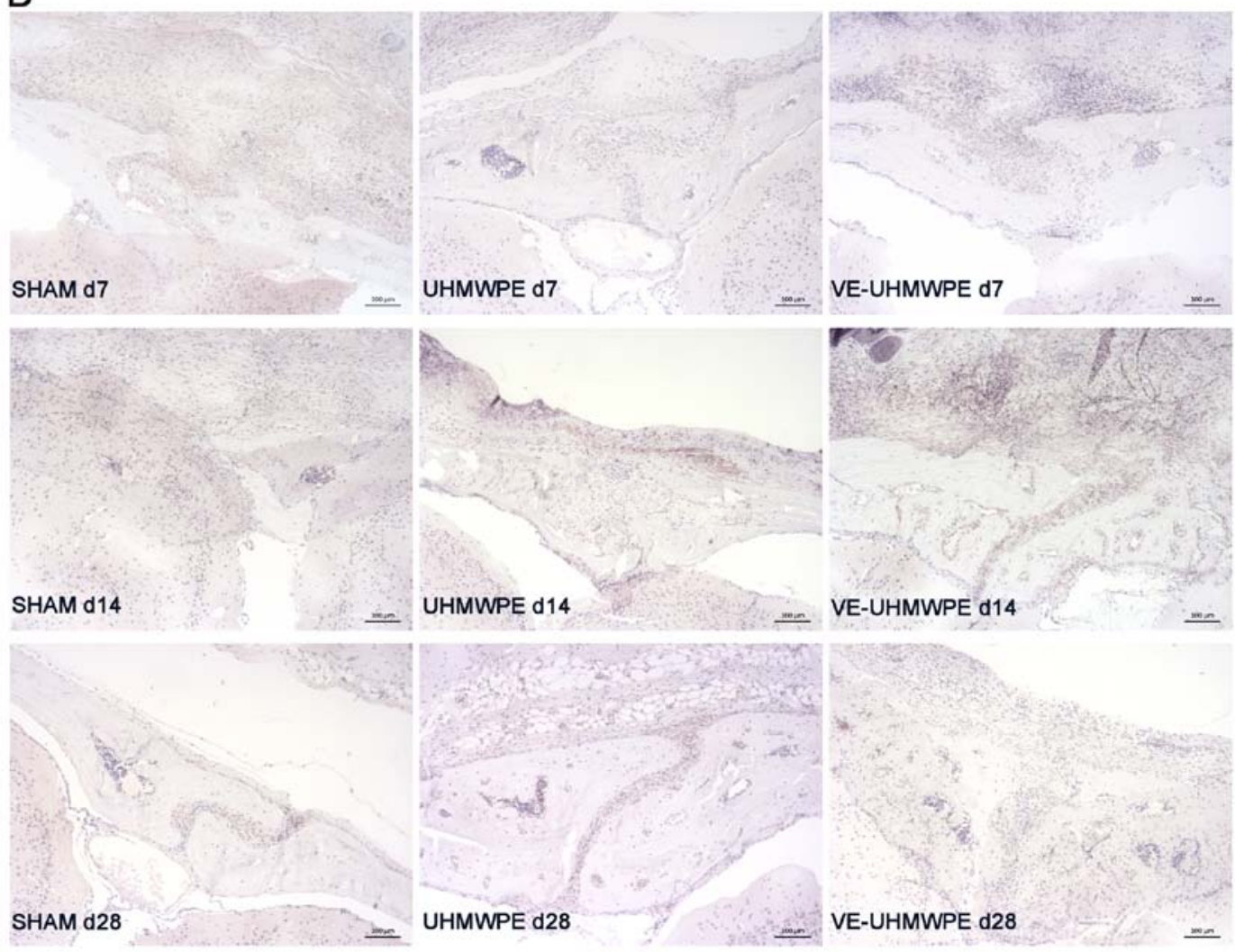

Figure 4.(A) Graphical illustration of mean serum $\alpha$-calcitonin gene-related peptide ( $\alpha$-CGRP)-levels + SD of each group, detected by ELISA. (B) Representative $\alpha$-CGRP antibody-stained sections of calvarias of mice at a magnification set at x10. The midline suture is located in the center. (C) Negative and positive controls of the spleen are demonstrated. d, day.

A

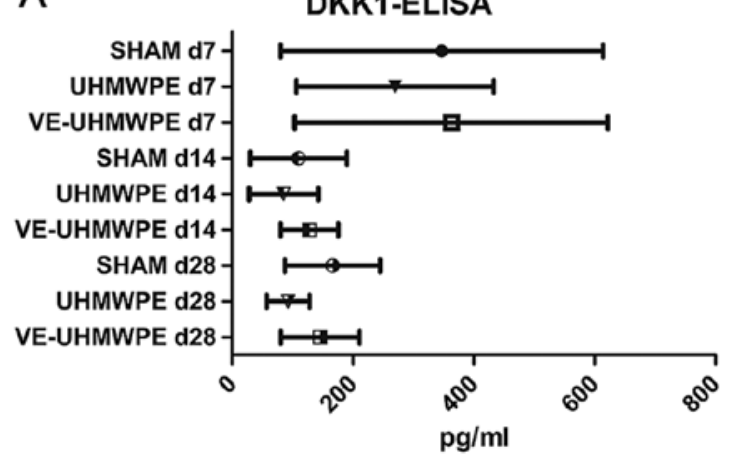

B

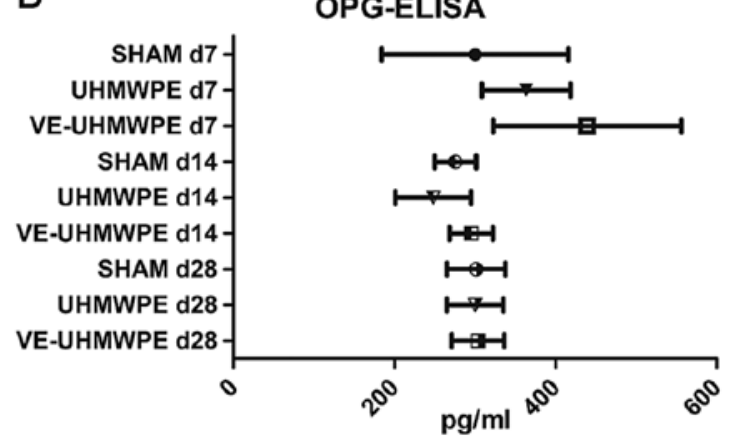

Figure 5. Markers of bone resorption [(DKK-1 (A)] and bone formation [OPG (B)] detected within the different groups at the end of the experimental time. Data are expressed as the means $+\mathrm{SD}$. d, day.

of the VE-UHMWPE particle-treated mice ( $\mathrm{p}=0,999$; Fig. 5A). Generally, the DKK-1 levels were lowest in all groups of mice at the experimental time of 14 days followed by a minor increase in DKK-1 levels in the animals treated at 28 days. Intergroup analysis, however, revealed no significant differences between any of the groups. 
Similar to the DKK-1 levels, the levels of the marker of bone formation, OPG, were higher in all animals treated at 7 days compared to the experimental time of 14 days (Fig. 5B). Although this difference was only significant in the UHMWPE and VE-UHMWPE-particle treated mice $(\mathrm{p}=0.005)$, there were no significant differences between the different groups. In the treated animals at 28 days, there was a trend towards increased OPG levels in all groups, yet there was not statistically significant difference.

\section{Discussion}

The biotribological performance of implants in endoprosthetic surgery is of superior importance as it contributes to the stability and longevity of implant anchorage. Therefore, the present study investigated the impact of VE-UHMWPE wear particles on the osseous microenvironment compared to UHMWPE wear particles.

In both the UHMWPE and VE-UHMWPE particle-treated groups, significant signs of particle-induced osteolysis were observed in the animals at 7 and 14 days compared to their corresponding controls (SHAM group). Of note, in the treated mice at 28 days, an increase in BV/TV was observed in both the UHMWPE and VE-UHMWPE particle-treated mice compared to the mice in the SHAM group, which could be attributed by a reactive osteoinduction following an increased inflammation in the early post-operative phase. In the present study, no significant differences in osteolysis were observed between the UHWMPE and VE-UHMWPE particle-treated mice, which is contradictory to the results reported by Bichara et al, who recently found a reduced osteolytic potential in VE-UHMWPE particle-treated mice compared to the controls (23). Another study on that topic published by Huang et al within this year also found similar signs of osteolysis between mice treated with vitamin E-containing particles and conventional UHMWPE wear particles (24). However, both studies investigated a murine calvaria model at a distinct time-point (10/14 days). Furthermore, Bichara et al investigated very young animals at the age of 8 weeks, whereas Huang et al used wear particles generated from wearing simulators; thus, the observed effect is likely to differ from PIO as it is typically seen. The use of an established murine calvaria model with different experimental duration times and more detailed immunohistochemical investigations may provide further insight. Diverging results may be attributed to the size of particles implanted, as the particle size is known to have a significant impact on the extent of the biological reaction to wear debris (25). Wear particles in the phagocytosable range from 0.3 to $10 \mu \mathrm{m}$ have been associated with the highest biological activity in in vitro studies (26). Studies on wear particles from retrieved human periprosthetic tissues and worn polyethylene surfaces are consistent with an average particle size in the range of $0.5 \mu \mathrm{m}$ in diameter (27). However, the ECD of the wear particles used in the present study was similar to the size of particles used in the experiments conducted by Bichara et al and Huang et al. Apart from the particle size, differences in reactivity may also be associated with the composition of wear particles. Thus, Bichara et al and Huang et al used cross-linked UHMWPE particles in their control group, whereas conventional UHMWPE particles were used in the present study, while differences in reactivity of conventional vs. cross-linked UHMWPE particles remain a matter of discussion (28). In addition, the amount of wear particles may have attributed to the extent of osteolysis observed in the UHMWPE and VE-UHMWPE particle-treated mice in the present study compared to the mice in the SHAM group. Thus, as an attempt to provoke a severe inflammatory response and to determine the osteolytic potential of UHMWPE and VE-UHMWPE particles, an extremely high concentration of particles was chosen in the present study, which was adapted to the amount of wear used in previous experiments by our group $(18,19)$. Huang et al treated the calvaria with $1 \mathrm{mg}$ of wear particles, Bichara et al chose a concentration of $3 \mathrm{mg}$, whereas a 30- to 10-fold higher particle concentration was chosen in the present study. In hip arthroplasty, a $28-\mathrm{mm}$ head with linear wear of $0.05 \mathrm{~mm} / \mathrm{y}$ corresponds to a volumetric wear rate of $30 \mathrm{~mm}^{3} / \mathrm{y}(27)$.

Therefore, a closer investigation of the osseous microenvironment and bone turnover may provide further insight into the biological activity of VE-UHMWPE wear particles in comparison to UHMWPE particles. As expected, the inflammatory response was pronounced in the early post-operative stage given the higher concentrations of TNF- $\alpha$ observed in all mice at day 7, accompanied by an increased number of osteoclasts. In the particle-treated mice, these appearances were associated with significant osteolysis, as indicated by a reduction in $\mathrm{BV} / \mathrm{TV}$. These data are supported by prior investigations on the inflammatory response of polyethylene particles. For example, Takahashi et al, using a luminescence murine calvaria model in $\mathrm{NF}-\kappa \mathrm{B} /$ luciferase transgenic mice, found that the level of luminescence was maximal at day 7 associated with a significant correlation with pro-inflammatory mediator mRNAs and bone resorption parameters (29). As TNF- $\alpha$ has been shown to be one of the key factors in the process of osteoclastogenesis, the use of anti-TNF agents may be a useful regenerative approach in the treatment of joint inflammation related to osteolysis (30). Whereas investigations on the biological reaction to wear particles have found a minor inflammatory response of ceramic wear particles compared to polyethylene wear particles (31), the present study revealed no potential differences in the inflammatory response in between UHMWPE and VE-UHMWPE wear particles.

Given the findings of $\alpha$-CGRP in the synovial fluid and periarticular tissue of loosened implants and the modulatory effect of vitamin $E$ on CGRP-based neuropeptides known from different experimental setups, further investigations in the present study aimed to identify the impact of VE-UHMWPE wear particles on the local neurogenic environment. Thus, biomarker analysis of serum $\alpha$-CGRP showed a continuous increase in $\alpha$-CGRP levels throughout the experimental duration time in all groups (SHAM, UHMWPE and VE-UHMWPE) and significantly higher $\alpha$-CGRP levels in the VE-UHMWPE particle-treated mice at the experimental time of 28 days, whereas minor $\alpha$-CGRP antibody concentrations were detectable for the first time via immunohistochemistry at day 14 in the UHMWPE and VE-UHMWPE particle-treated mice. Recent investigations by our group on haploinsufficient calcitonin receptor knockout mice identified that regenerative strategies in the aseptic loosening of endoprosthetic implants should focus on the impact of $\alpha$-CGRP-mediated signalling, although previous results have to be considered (18). Thus, 
experiments on $\alpha$-CGRP-deficient mice have demonstrated reduced PIO associated with decreased RANKL mRNA levels compared to wild-type controls, presuming a catabolic effect of $\alpha$-CGRP in particle induced osteolysis (19). On the contrary, in vitro analysis observed a time-dependent inhibitory effect of $\alpha$-CGRP on the secretion of osteolysis-associated pro-inflammatory cytokines such as TNF- $\alpha$ (32). Therefore, the observed increase in $\alpha$-CGRP levels found in the present study in the VE-UHMWPE particle-treated mice after a period of 28 days compared to the sham-operated controls has to be interpreted with caution. Although there is evidence, that $\alpha$-CGRP has a modulatory effect on the production of pro-inflammatory cytokines, its impact is not yet fully understood and there are limited data on the interaction between vitamin $\mathrm{E}$ and CGRP. As stated above, Tashima et al found that vitamin E supplementation provided a neuroprotective effect associated with a reduction in number of CGRP nerve fiber varicosities in the jejunum in experimental diabetes (13), although transmission of this interaction to the process of PIO involved uncertainties. Furthermore, the authors did not specify whether they detected $\alpha$-CGRP or $\beta$-CGRP nerve fibres, which differ from each other.

The investigation of bone turnover may therefore be an opportunity to further identify the impact of VE-UHMWPE particles in the process of PIO. It is well known that osteoclast and osteoblast activities in PIO are mediated by various inflammatory cytokines released from macrophages following the phagocytosis of wear particles (33). Therefore, differences in markers of bone formation and bone resorption would be expected if there were differences in the inflammatory response between UHMWPE and VE-UHMWPE wear particles. In the present study, significantly higher levels of DKK-1, a marker of bone resorption, were found in all mice after a period of 7 days compared to serum levels detected after a period of 14 days. Serum analysis similarly revealed significantly higher levels of OPG, a marker of bone formation, in particle-treated mice after a period of 7 days compared to the controls at 14 days. Taken together, no significant differences with regards to the inflammatory potential were observed between the UHMWPE und VE-UHMWPE particle-treated mice. These data are in accordance with previous in vitro testing comparing the biocompatibility of UHMWPE and vitamin E ( $\alpha$-tocopherol)stabilised UHMWPE tablets, in which similar proliferation rates were found in both polyethylene samples with no evidence of cytotoxicity (34).

In conclusion, the investigation of a very high concentration of VE-UHMWPE wear particles in an established murine calvaria model revealed a comparable extent of PIO as observed in UHMWPE wear particles. Only minor differences between the VE-UHMWPE particle- compared to the UHMWPE particle-treated mice were found with regards to an activation of inflammatory cytokines and osteoclasts, which was most distinct in the early stages after surgery. The impact of increased $\alpha$-CGRP levels observed in the mice treated with the VE-UHMWPE particles at 28 days and the influence of vitamin $\mathrm{E}$ on CGRP requires further investigation. However, as there were no differences in bone turnover between UHMWPE and VE-UHMWPE particle-treated mice, vitamin E-blended UHMWPE particles appear to have a reasonable biocompatibility. Taking the improved data on the tribologic and mechanical properties associated with a reduction of released wear particles into account, and given the increased ageing resistance of vitamin E-blended UHMWPE particles, the stabilization of polyethylene-bearing couples with vitamin $E$ appears to be a promising approach $(12,35)$. Further investigations on different concentrations of VE-UHMWPE wear particles and the identification of potential regenerative strategies remain of interest to reduce the extent of wear particle induced osteolysis in future.

Study limitations. There are some constraints of the experimental setup of this study, thus a very high concentration of wear particles was chosen as an attempt to provoke an inflammatory response which does not resemble the amount of wear occurring in humans. Furthermore, animals chosen for the present study were at the age of 12 weeks, as these wild-type mice are used very frequently this provides better comparison with other data, yet it has been shown that there are potential differences in bone metabolism of younger and aged mice (36). Also there may have been compensatory effects influencing the inflammatory response observed in the present model of particle induced osteolysis which could have been induced by other cytokines or peptide hormones that have not been detected.

\section{Acknowledgements}

The authors wish to thank Dr. A. Riedasch for support during the experimental time in the laboratory of animal science. Also many thanks to Mr. W. Wilfert at the Institute of Laboratory Medicine for the implementation of biomarker analysis. This study was supported by a research grant provided by the Friedrich-Baur-Foundation (FBS). The authors J. Schwiesau and T.M. Grupp are employes of the Aesculap AG.

\section{References}

1. Garellick G, Kärrholm J, Rogmark C, Rolfson O and Herberts P: Swedish Hip Arthroplasty Register, Annual Report 2011. Gothenburg, Sweden, 2012. ISBN 978-91-980507-1-4.

2. Jiang J, Yang CH, Lin Q, Yun XD and Xia YY: Does arthroplasty provide better outcomes than internal fixation a mid- and long-term followup? A meta-analysis. Clin Orthop Relat Res 473: 2672-2679, 2015.

3. Learmonth ID, Young $\mathrm{C}$ and Rorabeck $\mathrm{C}$ : The operation of the century: Total hip replacement. Lancet 370: 1508-1519, 2007.

4. Drees P, Eckardt A, Gay RE, Gay S and Huber LC: Molecular pathways in aseptic loosening of orthopaedic endoprosthesis. Biomed Tech (Berl) 53: 93-103, 2008 (In German).

5. Rubash HE, Sinha RK, Shanbhag AS and Kim SY: Pathogenesis of bone loss after total hip arthroplasty. Orthop Clin North Am 29: 173-186, 1998.

6. Schmalzried TP, Jasty M and Harris WH: Periprosthetic bone loss in total hip arthroplasty. Polyethylene wear debris and the concept of the effective joint space. J Bone Joint Surg Am 74: 849-863, 1992.

7. Grupp TM, Utzschneider S and Wimmer MA: Biotribology in knee arthroplasty. BioMed Res Int 2015: 618974, 2015.

8. Muratoglu OK, Bragdon CR, O'Connor DO, Jasty M and Harris WH: A novel method of cross-linking ultra-high-molecularweight polyethylene to improve wear, reduce oxidation, and retain mechanical properties. Recipient of the 1999 HAP Paul Award. J Arthroplasty 16: 149-160, 2001.

9. Thomas GE, Simpson DJ, Mehmood S, Taylor A, McLardy-Smith P, Gill HS, Murray DW and Glyn-Jones S: The seven-year wear of highly cross-linked polyethylene in total hip arthroplasty: A double-blind, randomized controlled trial using radiostereometric analysis. J Bone Joint Surg Am 93: 716-722, 2011. 
10. Oral E, Godleski Beckos C, Malhi AS and Muratoglu OK The effects of high dose irradiation on the cross-linking of vitamin E-blended ultrahigh molecular weight polyethylene. Biomaterials 29: 3557-3560, 2008.

11. Oral E, Wannomae KK, Rowell SL and Muratoglu OK: Diffusion of vitamin $\mathrm{E}$ in ultra-high molecular weight polyethylene. Biomaterials 28: 5225-5237, 2007.

12. Schwiesau J, Fritz B, Kutzner I, Bergmann G and Grupp TM: CR TKA UHMWPE wear tested after artificial aging of the vitamin $\mathrm{E}$ treated gliding component by simulating daily patient activities. BioMed Res Int 2014: 567374, 2014.

13. Tashima CM, Hermes-Uliana C, Perles JV, de Miranda Neto MH and Zanoni JN: Vitamins C and E (ascorbate/ $\alpha$-tocopherol) provide synergistic neuroprotection in the jejunum in experimental diabetes. Pathophysiology 22: 241-248, 2015.

14. Qian Y, Zeng BF, Zhang XL and Jiang Y: High levels of substance $\mathrm{P}$ and CGRP in pseudosynovial fluid from patients with aseptic loosening of their hip prosthesis. Acta Orthop 79: 342-345, 2008

15. Saxler G, Löer F, Skumavc M, Pförtner J and Hanesch U: Localization of SP- and CGRP-immunopositive nerve fibers in the hip joint of patients with painful osteoarthritis and of patients with painless failed total hip arthroplasties. Eur J Pain 11: 67-74, 2007.

16. Schinke T, Liese S, Priemel M, Haberland M, Schilling AF, CatalaLehnen P, Blicharski D, Rueger JM, Gagel RF, Emeson RB and Amling M: Decreased bone formation and osteopenia in mice lacking alpha-calcitonin gene-related peptide. J Bone Miner Res 19: 2049-2056, 2004

17. Kauther MD, Neuerburg C, Wefelnberg F, Bachmann HS, Schlepper R, Hilken G, Broecker-Preuss M, Grabellus F, Schilling AF, Jäger M and Wedemeyer C: RANKL-associated suppression of particle-induced osteolysis in an aged model of calcitonin and $\alpha$-CGRP deficiency. Biomaterials 34: 2911-2919, 2013.

18. Neuerburg C, Wedemeyer C, Goedel J, Schlepper R, Hilken G, Schwindenhammer B, Schilling AF, Jäger M and Kauther MD: The role of calcitonin receptor signalling in polyethylene particleinduced osteolysis. Acta Biomater 14: 125-132, 2015.

19. Wedemeyer C, Neuerburg C, Pfeiffer A, Heckelei A, Bylski D, von Knoch F, Schinke T, Hilken G, Gosheger G, von Knoch M, et al: Polyethylene particle-induced bone resorption in alphacalcitonin gene-related peptide-deficient mice. J Bone Miner Res 22: 1011-1019, 2007.

20. Utzschneider S, Becker F, Grupp TM, Sievers B, Paulus A, Gottschalk $\mathrm{O}$ and Jansson V: Inflammatory response against different carbon fiber-reinforced PEEK wear particles compared with UHMWPE in vivo. Acta Biomater 6: 4296-4304, 2010.

21. Standard Practice for Characterization of Particles. American Society for Testing and Materials, West Conshohocken, 2010.

22. Wedemeyer C, Xu J, Neuerburg C, Landgraeber S, Malyar NM, von Knoch F, Gosheger G, von Knoch M, Löer F and Saxler G: Particle-induced osteolysis in three-dimensional microcomputed tomography. Calcif Tissue Int 81: 394-402, 2007

23. Bichara DA, Malchau E, Sillesen NH, Cakmak S, Nielsen GP and Muratoglu OK: Vitamin E-diffused highly cross-linked UHMWPE particles induce less osteolysis compared to highly cross-linked virgin UHMWPE particles in vivo. J Arthroplasty 29 (Suppl): 232-237, 2014.
24. Huang $\mathrm{CH}$, Lu YC, Chang TK, Hsiao IL, Su YC, Yeh ST, Fang HW and Huang $\mathrm{CH}$ : In vivo biological response to highly cross-linked and vitamin e-doped polyethylene - a particleInduced osteolysis animal study. J Biomed Mater Res B Appl Biomater 104: 561-567, 2016.

25. Gallo J, Slouf M and Goodman SB: The relationship of polyethylene wear to particle size, distribution, and number: A possible factor explaining the risk of osteolysis after hip arthroplasty. J Biomed Mater Res B Appl Biomater 94: 171-177, 2010.

26. Green TR, Fisher J, Stone M, Wroblewski BM and Ingham E: Polyethylene particles of a 'critical size' are necessary for the induction of cytokines by macrophages in vitro. Biomaterials 19: 2297-2302, 1998.

27. Bezwada HP, Nazarian D and Booth R: Acetabular wear in total hip arthroplasty. E-Medicine.com: 1-15, 2004.

28. Catelas I, Wimmer MA and Utzschneider S: Polyethylene and metal wear particles: Characteristics and biological effects. Semin Immunopathol 33: 257-271, 2011.

29. Takahashi K, Onodera S, Tohyama H, Kwon HJ, Honma K and Yasuda K: In vivo imaging of particle-induced inflammation and osteolysis in the calvariae of $\mathrm{NF} \kappa \mathrm{B} /$ luciferase transgenic mice. J Biomed Biotechnol 2011: 727063, 2011.

30. Dong L, Wang R, Zhu YA, Wang C, Diao H, Zhang C, Zhao J and Zhang J: Antisense oligonucleotide targeting TNF- $\alpha$ can suppress Co-Cr-Mo particle-induced osteolysis. J Orthop Res 26: 1114-1120, 2008

31. Warashina H, Sakano S, Kitamura S, Yamauchi KI, Yamaguchi J, Ishiguro $\mathrm{N}$ and Hasegawa Y: Biological reaction to alumina, zirconia, titanium and polyethylene particles implanted onto murine calvaria. Biomaterials 24: 3655-3661, 2003.

32. Jablonski H, Kauther MD, Bachmann HS, Jager M and Wedemeyer C: Calcitonin gene-related peptide modulates the production of pro-inflammatory cytokines associated with periprosthetic osteolysis by THP-1 macrophage-like cells. Neuroimmunomodulation 22: 152-165, 2015.

33. Landgraeber S, Jäger M, Jacobs JJ and Hallab NJ: The pathology of orthopedic implant failure is mediated by innate immune system cytokines. Mediators Inflamm 2014: 185150, 2014.

34. Wolf C, Lederer K, Pfragner R, Schauenstein K, Ingolic E and Siegl V: Biocompatibility of ultra-high molecular weight polyethylene (UHMW-PE) stabilized with alpha-tocopherol used for joint endoprostheses assessed in vitro. J Mater Sci Mater Med 18: 1247-1252, 2007.

35. Bracco P and Oral E: Vitamin E-stabilized UHMWPE for total joint implants: A review. Clin Orthop Relat Res 469: 2286-2293, 2011.

36. Langlois J, Zaoui A, Bichara DA, Nich C, Bensidhoum M, Petite H, Muratoglu OK and Hamadouche M: Biological reaction to polyethylene particles in a murine calvarial model is highly influenced by age. J Orthop Res 34: 574-580, 2016. 